\title{
In vitro chemotactic responses of Brugia pahangi infective larvae to sodium ions
}

\author{
Y. Mitsui ${ }^{1 *}$, M. Miura ${ }^{1}$, D.A. Bome ${ }^{2}$ and Y. Aoki ${ }^{1}$ \\ ${ }^{1}$ Department of Parasitology, Institute of Tropical Medicine, Nagasaki \\ University, 1-14-2 Sakamoto, Nagasaki 852-8523, Japan: ${ }^{2}$ Kailahun \\ Government Hospital, Kailahun District, Sierra Leone
}

(Received 23 February 2011; Accepted 26 September 2011; First Published Online 27 October 2011)

\begin{abstract}
In vitro chemotactic responses of infective third-stage larvae (L3) of Brugia pahangi to $\mathrm{NaCl}, \mathrm{Na}_{2} \mathrm{HPO}_{4}, \mathrm{KCl}, \mathrm{K}_{2} \mathrm{HPO}_{4}, \mathrm{MgCl}_{2}$ and $\mathrm{CaCl}_{2}$ were assessed. Compared to deionized water as a control, $200 \mathrm{mM} \mathrm{NaCl}$ and $100 \mathrm{mM} \mathrm{Na} \mathrm{NPO}_{4}$ significantly attracted L3 $(P<0.01$ and $P<0.01)$, whereas L3 were likely to avoid $200 \mathrm{~mm} \mathrm{KCl}$ and $100 \mathrm{mM} \mathrm{K} \mathrm{HPO}_{4}(P<0.05$ and $P<0.05)$. L3 showed no significant tendency to avoid or to be attracted to $200 \mathrm{mM} \mathrm{CaCl}$ and $200 \mathrm{~mm}$ $\mathrm{MgCl}_{2}$. Furthermore, $\mathrm{NaCl}$ exhibited a significant chemoattractant activity for $\mathrm{L} 3$ at a low concentration of $100 \mathrm{~mm}$.
\end{abstract}

\section{Introduction}

The chemotaxis of Caenorhabditis elegans, a free-living nematode, was first described by Ward (1973). Several salts, some amino acids and some nucleotides were identified as water-soluble attractants for C. elegans. With regard to skin-penetrating parasitic nematodes, a component of the host blood is related to the host finding and penetrating behaviours of their infective third-stage larvae (L3). Wauters et al. (1982) and Vetter et al. (1985) indicated that dog serum contained an attractant for the L3 of the hookworm Ancylostoma caninum. Subsequently, Tobata-Kudo et al. (2000) and Forbes et al. (2003) revealed that sodium chloride, a major component of serum, was one of the chemoattractants for the L3 of the threadworms Strongyloides ratti and S. stercoralis, respectively.

Although filarial worms are one of several skinpenetrating helminths, the penetration behaviour of filarial L3 is unlike that of the L3 of hookworms and threadworms. The L3 of hookworms can penetrate the intact skin of hosts directly and invade the body of a host (Vetter \& van der Linden, 1977). On the other hand, when infected mosquitoes feed on a host, filarial L3 emerge from the proboscis and lie on the skin surface of the host (Ewert, 1967; Ewert \& Ho, 1967). They presumably then move towards the bite wound made by the mosquito and penetrate the skin via the wound. It is easily

*Fax: + 81-95-819-7824

E-mail: ymitsui@nagasaki-u.ac.jp surmised that a component of the host blood affects the movements of filarial L3 towards the bite wound.

A recent study (Gunawardena et al., 2003) revealed that the filarial L3 of Brugia pahangi, a skin-penetrating parasitic nematode, were highly attracted to the serum of Mongolian jird (Meriones unguiculatus). In addition, Kusaba et al. (2008) showed that the sera derived from various mammals attracted filarial L3. These results suggested that the sera contained an attractant for the L3. Thus, sodium ions, a major component of serum, have been regarded as a promising attractant of the L3. Nevertheless, no attempt has yet been made to assess the chemoattractant activity of sodium ions for filarial L3, even though identification of the chemical attractant is an important prerequisite for understanding the mechanisms of skin-penetrating infection of filarial L3.

The objective of the present study was to investigate the chemotactic reactivity of sodium chloride for B. pahangi $\mathrm{L} 3$ using the modified method of Gunawardena et al. (2003).

\section{Materials and methods}

Chemicals

Hanks' balanced salt solution (HBSS) was purchased from Nissui Pharmaceutical Co. Ltd (Tokyo, Japan). Fetal bovine serum (FBS) was purchased from Gibco (Langley, Oklahoma, USA). $\mathrm{NaCl}, \mathrm{Na}_{2} \mathrm{HPO}_{4}, \mathrm{KCl}$, $\mathrm{K}_{2} \mathrm{HPO}_{4}, \mathrm{CaCl}_{2}$ and $\mathrm{MgCl}_{2}$ were purchased from Wako 
Pure Chemical Industries, Ltd (Osaka, Japan). All the other chemicals and salts were of analytical grade.

\section{Parasite strain}

The filarial parasite B. pahangi used in the present experiment had been maintained in Mongolian jirds (M. unguiculatus) and Aedes aegypti (Liverpool strain) mosquitoes in the Animal Research Center for Tropical Infections at the Institute of Tropical Medicine, Nagasaki University. Brugia pahangi L3 were harvested from mosquitoes that had been fed on microfilaraemic jirds 2 weeks previously. The infected mosquitoes were dissected in HBSS thereafter. L3 were collected and washed twice in HBSS prior to assays of the chemotactic responses of L3.

The experimental protocol was approved by the Animal Care and Use Committee, Nagasaki University. Animal care and experimental procedures were performed in accordance with the Guidelines for Animal Experimentation of Nagasaki University.

\section{Assay using fetal bovine serum and salts}

The chemotactic responses of filarial L3 to FBS and salts were measured on agar plates according to a modified method previously described by Gunawardena et al. (2003). Briefly, a 35-mm Petri dish (Sumilon, Sumitomo Bakelite Co. Ltd, Tokyo, Japan) was filled with $2 \mathrm{ml}$ of $0.6 \%(\mathrm{w} / \mathrm{v})$ Noble agar (Difco Laboratories, Inc., Detroit, Michigan, USA) dissolved in hot deionized water (DW) and allowed to cool at room temperature. The agar plate was placed over a template transparency sheet (fig. 1) on which three circles $3 \mathrm{~mm}$ in diameter were drawn to indicate areas where L3 (I-area), test solution (T-area) and DW ( $D$-area) were spotted. The sheet also contained two concentric circles of $10 \mathrm{~mm}$ each, indicating test solution $(T)$ and DW $(D)$ zones, and outside zone $(O)$ surrounding the $I$-area, the $T$ - and $D$-zones. One microlitre of DW was spotted at the I-area of an agar plate. Immediately, approximately ten L3 were placed into the $I$-area in the agar plate using a fine needle. Two microlitres of test solution containing FBS or test salts dissolved in DW at concentrations of 100 or $200 \mathrm{mM}$, and $2 \mu \mathrm{l}$ of DW were spotted on the right $(T)$ and left $(D)$ areas, respectively. Then the agar plate was placed on a hot plate (ND-1, Asone Corporation, Osaka, Japan) with temperature adjusted to $35^{\circ} \mathrm{C}$. Over a period of $60 \mathrm{~min}$ or after $30 \mathrm{~min}$, the number of L3 accumulating in the four sectors (I-area, $T$-, $D$ - and $O$-zones) was determined by counting under a dissecting microscope. The assays were repeated six times for each test solution. In the assay for responses of filarial L3 to $\mathrm{NaCl}, \mathrm{NaCl}$ was dissolved in DW at concentrations of 0 (control), 50, 100, 150, 200, 250, 300,350 and $400 \mathrm{~mm}$, and used as test solutions.

\section{Statistical analysis}

The proportion of filarial L3 accumulating in each sector was expressed as $100 \times$ (number of L3 in each sector) /(total number of L3 applied to the six agar plates). A $95 \%$ confidence interval $(95 \% \mathrm{CI})$ of the proportion was calculated according to the procedure described by Daniel (1999a). Furthermore, comparisons of the

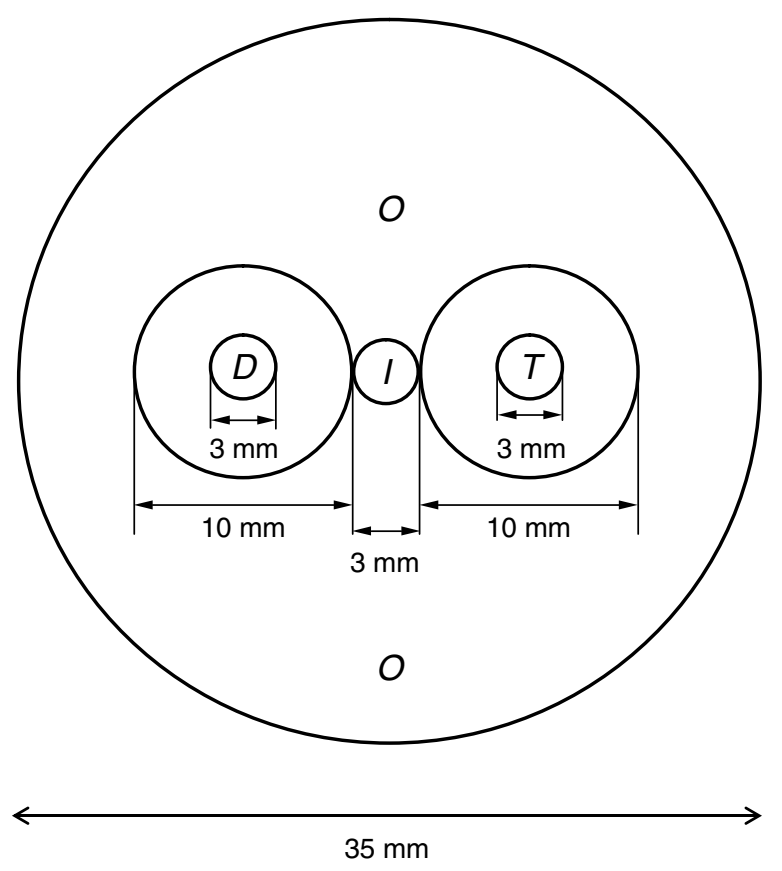

Fig. 1. Diagrammatic representation of the sectors used to quantify the larval response to test solutions. The small circles marked by $I, D$ and $T$, indicate the areas on to which $1 \mu \mathrm{l}$ of deionized water (DW) and infective third-stage larvae (I-area), $2 \mu \mathrm{l}$ of DW alone ( $D$-area) and test solution ( $T$-area) were placed, respectively. The large concentric circles were marked as $D$ - and $T$-zones, and the outside zone was marked as $O$.

proportions of L3 accumulating in the T-zone of a test salt solution and DW (control group) were conducted using the $z$-test (Daniel, 1999b).

\section{Results}

\section{Responses to fetal bovine serum}

The time course of the chemotactic response of filarial L3 to FBS is presented in fig. 2. About ten L3, FBS and DW were applied to the $I-, T$ - and $D$-areas of an agar plate, respectively, and the numbers of L3 accumulating in the four sectors ( $I$-area, $T$-, $D$ - and $O$-zones) were counted over a period of $60 \mathrm{~min}$. The assays were repeated six times. Figure 2 shows the proportion of L3 accumulating in each sector at 10, 20,30, 40 and $60 \mathrm{~min}$. At $10 \mathrm{~min}$ after the application of L3, FBS and DW to the agar plate, $82 \%$ of L3 remained in the $I$-area, while $18 \%$ of L3 moved toward the $D$-, $T$ - or O-zone. At $20 \mathrm{~min}$, only $18 \%$ of $\mathrm{L} 3$ remained in the $I$-area, while $73 \%$ of L3 had moved to the T-zone of FBS. At $30 \mathrm{~min}$, the proportion of L3 accumulating in the T-zone reached the peak of $74 \%$. Subsequently, the proportion of L3 accumulating in the $T$-zone gradually decreased, and the proportion of L3 in the O-zone gradually increased up to $18 \%$ at $60 \mathrm{~min}$.

\section{Responses to test salts}

Table 1 shows the chemotactic response of L3 to salts: $\mathrm{NaCl}, \mathrm{Na}_{2} \mathrm{HPO}_{4}, \mathrm{KCl}, \mathrm{KH}_{2} \mathrm{PO}_{4}, \mathrm{CaCl}_{2}$ and $\mathrm{MgCl}_{2}$. 


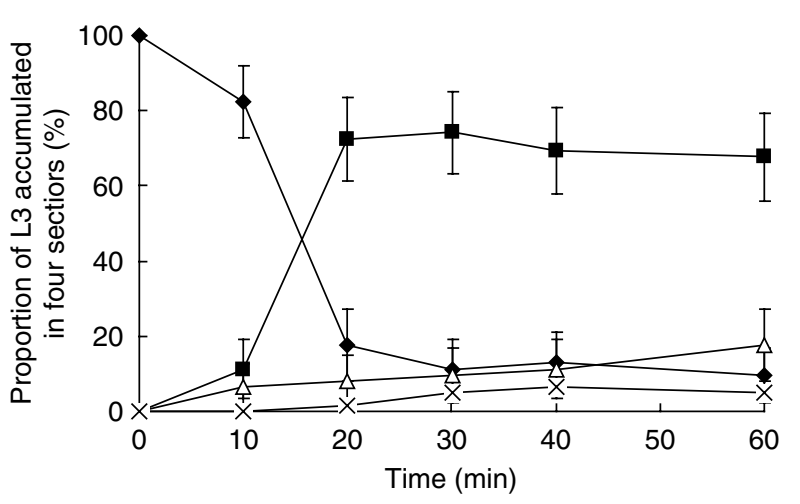

Fig. 2. The time course of the chemotactic response of infective third-stage larvae (L3) of Brugia pahangi to fetal bovine serum (FBS). Four sectors are indicated as follows: $\bullet, I$-area; $\mathbf{\square}$, FBS-zone; $\triangle, O$-zone; $\times, D$-zone. Each error bar shows the $95 \%$ confidence interval for the proportion of L3 accumulating in each sector.

The accumulation of L3 in the T-zone of test salt solutions was observed $30 \mathrm{~min}$ after about ten L3, test salt solution and DW were applied to an agar plate. When DW was applied to $T$ - and $D$-areas as the control group, $17 \%$ of L3 moved to the T-zone. Meanwhile, $69 \%$ and $86 \%$ of L3 accumulated in the T-zone of $200 \mathrm{~mm} \mathrm{NaCl}$ and $100 \mathrm{~mm}$ $\mathrm{Na}_{2} \mathrm{HPO}_{4}$, respectively. Both $200 \mathrm{~mm} \mathrm{NaCl}$ and $100 \mathrm{~mm}$ $\mathrm{Na}_{2} \mathrm{HPO}_{4}$ attracted L3 at a significantly higher level than DW $(P<0.01$ and $P<0.01)$. On the contrary, the L3 were likely to avoid $200 \mathrm{mM} \mathrm{KCl}$ and $100 \mathrm{mM} \mathrm{K}_{2} \mathrm{HPO}_{4}(P<0.05$ and $P<0.05)$. L3 showed no significant tendency to avoid or to be attracted to $200 \mathrm{mM} \mathrm{CaCl}_{2}$ and $200 \mathrm{mM} \mathrm{MgCl}_{2}$.

\section{Responses to sodium chloride}

The chemotactic response of $\mathrm{L} 3$ to $\mathrm{NaCl}$ was observed in the concentration range of $0 \mathrm{~mm}$ to $400 \mathrm{~mm}$ (fig. 3). The accumulation of $\mathrm{L} 3$ in a $\mathrm{T}$-zone of $\mathrm{NaCl}$ was observed 30 min after about ten $\mathrm{L} 3, \mathrm{NaCl}$ solution and $\mathrm{DW}$ were applied to an agar plate. The assays were repeated six times for each concentration of $\mathrm{NaCl}$. When $\mathrm{NaCl}$ was applied to the $T$-area at a concentration of $50 \mathrm{~mm}, 23 \%$

Table 1. Chemotactic responses of infective third-stage larvae (L3) of Brugia pahangi to different salts.

\begin{tabular}{lccc}
\hline $\begin{array}{l}\text { Test salt solution } \\
\text { and their } \\
\text { concentrations }\end{array}$ & $\begin{array}{c}\text { Total number of } \\
\text { L3 applied to } \\
\text { six agar plates }\end{array}$ & $\begin{array}{c}\text { Percent proportion } \\
\text { of L3 accumulating } \\
\text { in T-zone }(95 \% \mathrm{CI})\end{array}$ & $\begin{array}{c}P \\
\text { value }^{\text {a }}\end{array}$ \\
\hline Deionized water & 60 & $16.7(7.2-26.1)$ & - \\
$200 \mathrm{mM} \mathrm{NaCl}$ & 61 & $68.9(57.2-80.5)$ & $<0.01$ \\
$100 \mathrm{mM} \mathrm{Na} \mathrm{HPO}_{4}$ & 62 & $85.5(76.7-94.3)$ & $<0.01$ \\
$200 \mathrm{mM} \mathrm{KCl}$ & 60 & $3.3(0-7.9)$ & $<0.05$ \\
$100 \mathrm{mM} \mathrm{K}_{2} \mathrm{HPO}_{4}$ & 63 & $4.8(0-10.0)$ & $<0.05$ \\
$200 \mathrm{mM} \mathrm{CaCl}_{2}$ & 59 & $8.5(1.4-15.6)$ & 0.18 \\
$200 \mathrm{mM} \mathrm{MgCl}_{2}$ & 59 & $28.8(17.3-40.4)$ & 0.11 \\
\hline
\end{tabular}

The number of L3 accumulating in each sector was counted $30 \mathrm{~min}$ after around $10 \mathrm{L3}$, deionized water (DW) and test solution were placed on an agar plate. The assays were repeated six times for each test solution.

${ }^{a}$ The proportion of L3 accumulating in the T-zone of each test salt was compared with that in DW (control) according to the z-test.

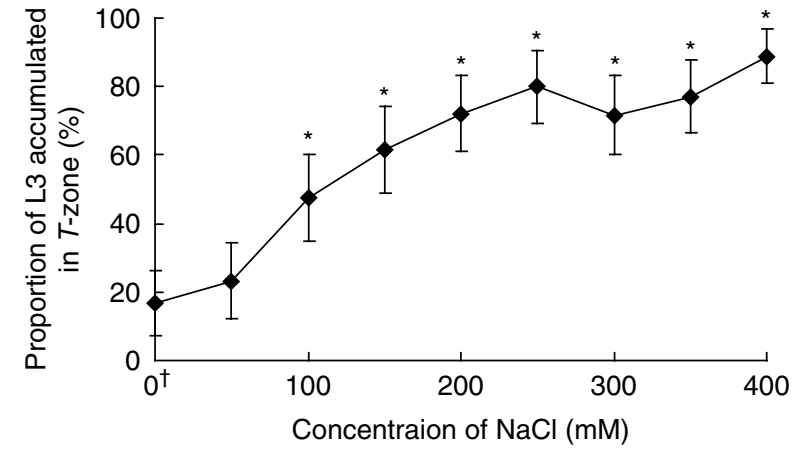

Fig. 3. The effect of $\mathrm{NaCl}$ concentration on the chemotactic responses of infective third-stage larvae (L3) of Brugia pahangi. Each error bar shows the $95 \%$ confidence interval for the proportion of L3 accumulating in the $T$-zone. ${ }^{*} P<0.01$, the proportion of $\mathrm{L} 3$ accumulating in the $\mathrm{T}$-zone of $\mathrm{NaCl}$ at different concentrations was compared with that of deionized water (DW), z-test. +Control (DW alone in the T-zone).

of L3 accumulated in the T-zone. The proportion of L3 accumulating in the T-zone was slightly higher than that with DW (17\%). At concentrations of more than $100 \mathrm{~mm}$, $\mathrm{NaCl}$ attracted L3 at a significantly higher level than DW $(P<0.01)$.

\section{Discussion}

The response of an organism to environmental changes is crucial to its survival. Parasitic nematodes of warmblooded hosts use chemical signals in host finding. Tobata-Kudo et al. (2000) and Forbes et al. (2003) revealed the chemotactic activity of $\mathrm{NaCl}$ for infective L3 of $S$. ratti and $S$. stercoralis, respectively. Filariae are also skin-penetrating parasitic nematodes, and their infection of hosts involves a chemotactic response. Recent studies (Gunawardena et al., 2003; Kusaba et al., 2008) have suggested that mammalian sera contain a chemoattractant for B. pahangi L3. Since these studies, however, there has been little progress in investigations regarding the attractants of B. pahangi L3.

The procedure previously described by Gunawardena et al. (2003) applied HBSS to the I-area where filarial L3 were inoculated (fig. 1). As a method for testing the chemotactic response of L3 to salts, HBSS was thought to be unsuitable, because it contained salts similar to those tested for the chemotactic response of L3. Thus, in the present study, DW alone was spotted on to an I-area instead of HBSS. In addition, the volume applied to the $I$-area was reduced from $2 \mu \mathrm{l}$ to $1 \mu \mathrm{l}$ to shorten the lingering time of L3 in the $I$-area, because a large amount of DW spotted in the area confined L3 to the I-area until the DW was either absorbed into the agar plate or evaporated (fig. 1). In the modified chemotaxis assay, the peak proportion of L3 accumulating in the T-zone of FBS was reached at $30 \mathrm{~min}$ after L3, FBS and DW were placed on the agar plate (fig. 2). On the other hand, the peak proportion of L3 accumulating in the T-zone of FBS by the chemotaxis assay of Gunawardena et al. (2003) was observed at $60 \mathrm{~min}$. After the application of L3 to the agar plate, the L3 were induced to move toward the T-zone 
of FBS earlier in the modified assay than in the assay of Gunawardena et al. (2003). In the modified chemotaxis assay, $30 \mathrm{~min}$ was considered the optimal time for observing the chemotactic response of $\mathrm{L} 3$ to salts.

$\mathrm{NaCl}, \mathrm{Na}_{2} \mathrm{HPO}_{4}, \mathrm{KCl}, \mathrm{K}_{2} \mathrm{HPO}_{4}, \mathrm{CaCl}_{2}$ and $\mathrm{MgCl}_{2}$ were examined for the chemotactic response of B. pahangi L3 (table 1). L3 were significantly more attracted to $200 \mathrm{~mm}$ $\mathrm{NaCl}(69 \%)$ and $100 \mathrm{~mm} \mathrm{Na} \mathrm{HPO}_{4}(86 \%)$ than to $\mathrm{DW}$ $(17 \%)$. These results suggest that sodium ions are one of the attractants of $B$. pahangi L3. Although Tobata-Kudo et al. (2000) and Forbes et al. (2003) revealed that sodium chloride was a chemoattractant of $S$. ratti and $S$. stercoralis L3, respectively, the chemotactic response of $\mathrm{L} 3$ to $\mathrm{NaCl}$ was observed when L3 were placed in a concentration of $\mathrm{NaCl}$ lower than $20 \mathrm{~mm}$ and $10 \mathrm{~mm}$. In general, the L3 of parasitic nematodes seem to recognize sodium ions as an attractant. Conversely, filarial L3 were likely to avoid $200 \mathrm{~mm} \mathrm{KCl}$ and $100 \mathrm{mM} \mathrm{K}_{2} \mathrm{HPO}_{4}$ (table 1), suggesting that potassium ions are a negative attractant to L3. On the other hand, L3 showed no significant tendency to avoid or to be attracted to $\mathrm{CaCl}_{2}$ and $\mathrm{MgCl}_{2}$. Sodium, potassium and magnesium ions are attractants of C. elegans, a free-living nematode (Ward, 1973), thus the chemotactic responses of filarial L3 to metal ions differed greatly from those of C. elegans.

The peak proportion of filarial L3 accumulating in the $\mathrm{T}$-zone of $\mathrm{NaCl}$ was dependent on $\mathrm{NaCl}$ concentration in the $\mathrm{T}$-zone up to $250 \mathrm{mM}$. $\mathrm{NaCl}$ significantly attracted the L3 at a lower concentration of $100 \mathrm{~mm}$, which is less than the concentration of sodium ions in human blood (130-150 mM). Thus, $100 \mathrm{~mm}$ might be a sufficient concentration for sodium ions to attract filarial L3 in natural infections.

Although the present study revealed the remarkable attraction of $B$. pahangi $\mathrm{L} 3$ to $\mathrm{NaCl}$, animal blood is composed not only of sodium ions but also other various substances. Thus, it is presumed that other substances, although as yet unidentified, can also exhibit chemoattractant activity for filarial L3. In addition, Safer et al. (2007) recently revealed that urocanic acid (UCA) was the chemoattractant for S. stercoralis, a skinpenetrating parasitic nematode. UCA is also expected to be the chemoattractant for B. pahangi L3. It is surmised that, in natural infection of a host, filarial L3 recognize not only sodium ions but also other substances of the host blood leaking from the wound caused by a mosquito bite and move to the wound before penetrating the skin. Besides substances of the host blood, other biological substances may be related to the migratory behaviour of filarial L3 on the skin of their natural hosts. Then, the finding that sodium ions are a chemoattractant of the L3 is the first step for an understanding of the mechanisms of skin-penetrating infection by the third-stage larvae.

\section{Acknowledgements}

This work was partially supported by the Japanese International Cooperation Agency (JICA) scholarship programme.

\section{References}

Daniel, W.W. (1999a) Confidence interval for a population proportion. pp. 176-178 in Daniel, W.W. (Ed.) Biostatistics: A foundation for analysis in the health sciences. 7th edn. New York, John Wiley \& Sons Inc.

Daniel, W.W. (1999b) Hypothesis testing: the difference between two population proportions. pp. 252-255 in Daniel, W.W. (Ed.) Biostatistics: A foundation for analysis in the health sciences. 7th edn. New York, John Wiley \& Sons Inc.

Ewert, A. (1967) Studies on the transfer of infective Brugia pahangi larvae from vector mosquitoes to the mammalian host. Transactions of the Royal Society of Tropical Medicine and Hygiene 61, 110-113.

Ewert, A. \& Ho, B.C. (1967) The fate of Brugia pahangi larvae immediately after feeding by infective vector mosquitoes. Transactions of the Royal Society of Tropical Medicine and Hygiene 61, 659-662.

Forbes, W.M., Ashton, F.T., Boston, R. \& Schad, G.A. (2003) Chemotactic behaviour of Strongyloides stercoralis infective larvae on a sodium chloride gradient. Parasitology 127, 189-197.

Gunawardena, N.K., Fujimaki, Y. \& Aoki, Y. (2003) Chemotactic response of Brugia pahangi infective larvae to jird serum in vitro. Parasitology Research 90, $337-342$.

Kusaba, T., Fujimaki, Y., Vincent, A.L. \& Aoki, Y. (2008) In vitro chemotaxis of Brugia pahangi infective larave to the sera and hemolymph of mammals and lower animals. Parasitology International 57, 179-184.

Safer, D., Brenes, M., Dunipace, S. \& Schad, G. (2007) Urocanic acid is a major chemoattractant for the skin-penetrating parasitic nematode Strongyloides stercoralis. Proceedings of the National Academy of Sciences of the United States of America 104, 1627-1630.

Tobata-Kudo, H., Higo, H., Koga, M. \& Tada, I. (2000) Chemokinetic behavior of the infective third-stage larvae of Strongyloides ratti on a sodium chloride gradient. Parasitology International 49, 183-188.

Vetter, J.C. \& van der Linden, M.E. (1977) Skin penetration of infective hookworm larvae. I. The path of migration of infective larvae of Ancylostoma braziliense in canine skin. Zeitschrift für Parasitenkunde 53, 255-262.

Vetter, J.C., Vingerhoed, J., Schoeman, E. \& Wauters, H.W. (1985) Chemotactic attraction of infective hookworm larvae of Ancylostoma caninum by a dog serum factor. Zeitschrift für Parasitenkunde 71, 539-543.

Ward, S. (1973) Chemotaxis by the nematode Caenorhabditis elegans: identification of attractants and analysis of the response by use of mutants. Proceedings of the National Academy of Sciences of the United States of America 70, 817-821.

Wauters, H.W., Klaver-Wesseling, J.C. \& Vetter, J.C. (1982) The effect of ultrafiltrated and dialysed dog serum on the chemotaxis of infective hookworm larvae of Ancylostoma caninum. Zeitschrift für Parasitenkunde 68, 305-311. 